\title{
Expression of the platelet-activating factor receptor enhances benzyl isothiocyanate-induced apoptosis in murine and human melanoma cells
}

\author{
RAVI PRAKASH SAHU ${ }^{1,2}$ \\ Departments of ${ }^{1}$ Pathology and Laboratory Medicine and ${ }^{2}$ Dermatology, \\ Indiana University School of Medicine, Indianapolis, IN 46202, USA
}

Received March 11, 2014; Accepted November 20, 2014

DOI: $10.3892 / \mathrm{mmr} .2015 .3371$

\begin{abstract}
Melanoma cells often express platelet-activating factor receptor (PAF-R), which has been demonstrated to increase metastatic behavior. However, the effect of PAF-R on the responsiveness of melanoma to naturally occurring cytotoxic agents remains to be elucidated. The present study aimed to determine the relative cytotoxicity and mechanism of benzyl isothiocyanate (BITC), a component of cruciferous vegetables, in melanoma cells expressing PAF-R. To evaluate the importance of PAF-R signaling in melanoma cell growth, PAF-R-negative murine B16F10 cells were transduced with a retrovirus containing the cDNA for PAF-R to generate cells stably expressing PAF-R (B16-PAF-R) or an empty vector (MSCV) to generate PAF-R-deficient B16-MSCV control cells. Activation of PAF-R, using the PAF-R agonist, 1-hexadecyl-2-N-methylcarbamoyl-3-glycerophosphocholine, induced an increase in the proliferation of B16-PAF-R cells compared with the B16-MSCV cells. Reverse transcription quantitative polymerase chain reaction revealed the presence of functional PAF-R in human melanoma SK23MEL cells, but not in SK5MEL cells. The present study investigated the effect of BITC treatments on the survival of murine and human melanoma cells, in the presence or absence of functional PAF-R. The results revealed that treatment with BITC decreased the survival rate of the PAF-R-positive and negative murine and human melanoma cells. However, the expression of PAF-R
\end{abstract}

Correspondence to: Ravi Sahu, Indiana University School of Medicine, IB419, 975 Walnut Street, Indianapolis, IN 46202, USA E-mail: rsahu@iupui.edu

Abbreviations: $\mathrm{PAF}$, platelet-activating factor; PAF-R,PAF-receptor; CPAF, 1-hexadecyl-2-N-methylcarbamoyl-3-glycerophosphocholine; BITC, benzyl isothiocyanate; ROS, reactive oxygen species; FACS, fluorescence-activated cell sorting; mRNA, messenger RNA; PBS, phosphate-buffered saline; RT-qPCR, reverse transcription-quantitative polymerase chain reaction

Key words: apoptosis, benzyl isothiocyanate, melanoma, platelet-activating factor-receptor, reactive oxygen species substantially augmented BITC-mediated cytotoxicity in the PAF-R-positive cells at lower concentrations compared with the PAF-R-negative cells. In order to determine the underlying mechanism, flow cytometric analysis was used, which demonstrated a significant increase in the generation of reactive oxygen species (ROS) in the B16-PAF-R cells compared with the B16-MSCV cells, which enhanced apoptosis by BITC, as measured by increased caspase-3/7 luminescence. Notably, the BITC-mediated decreased cell survival rate, increased ROS and increased apoptosis in the B16-PAF-R cells were significantly attenuated by the antioxidant, vitamin $\mathrm{C}$, indicating ROS involvement. Additionally, the WEB2086 PAF-R antagonist, inhibited the BITC-mediated enhancement of apoptosis in the B16-PAF-R cells, indicating a role for PAF-R-signaling in the BITC-mediated effects. These findings indicated that the selectivity of BITC towards PAF-R in melanoma offers a promising chemopreventive agent for PAF-R-positive melanoma treatment.

\section{Introduction}

The incidence of malignant melanoma is rapidly increasing, with the estimated annual mortality rate of $>9,000$ in the USA $(1,2)$. Melanoma cells often express receptors for multiple growth factors and cytokines, which regulate their growth, including platelet-activating factor-receptor (PAF-R), a G-protein coupled receptor expressed in various types of cell (3-5). The activation of PAF-R has been demonstrated to mediate diverse biological functions in response to various stimuli, including inflammation, immunosuppression and tumorigenesis (6-13).

Previous studies, including ours, have demonstrated that the activation of PAF-R positively modulates melanoma growth, either directly (9-12) or indirectly via host immunomodulation (13). However, in epithelial cell types, activation of PAF-R can increase cell death, induced by pro-oxidative stress reagents $(14,15)$. As melanoma is highly resistant to chemotherapy and radiation therapy (16), identifying novel pathways that can augment cytotoxic agent effects may offer a promising therapeutic approach for melanoma.

Isothiocyanates (ITCs), which are present in cruciferous vegetables, including broccoli and cabbage, possess anti-carcinogenic properties $(17,18)$. Benzyl isothiocyanate (BITC), an 
analog of ITC suppresses the in vitro and in vivo growth of various types of cancer (19-22). In melanoma, BITC and other isoforms of ITCs, including allyl and phenyl isothiocyanates and sulforaphane, have been observed to inhibit melanoma cell growth via different mechanisms (23-27). Since many melanomas express functional PAF-Rs and the role of PAF-R in the BITC-mediated suppression of melanoma cells remain to be elucidated, the present study aimed to assess whether the expression of PAF-R can augment the BITC-mediated cytotoxic effects in melanoma cells.

\section{Materials and methods}

Reagents. A Qiagen RNeasy Mini kit for RNA extraction was purchased from Qiagen Sciences (Germantown, MD, USA), and the Super Script (R) First-Strand Synthesis system for cDNA synthesis was purchased from Invitrogen Life Technologies, Carlsbad, CA, USA). The PAF-R and GAPDH primers and the SYBR Green polymerase chain reaction (PCR) reagents were purchased from SABiosciences (Valencia, CA, USA). A caspase-3/7 activity assay kit was purchased from Promega Corporation (Madison, WI, USA). The WEB2086 PAF-R antagonist, was purchased from Cayman Chemicals Co. (Ann Arbor, MI, USA). All other reagents were purchased from Sigma-Aldrich (St. Louis, MO, USA).

Cells. Murine B16 cells expressing PAF-R (B16-PAFR), empty vector (B16-MSCV) and human SK23MEL melanoma cells were maintained in RPMI-1640 media (Life Technologies, Grand Island, NY, USA) supplemented with $10 \%$ fetal bovine serum (HyClone, GE Healthcare Life Sciences, Logan, UT, USA) and $100 \mu \mathrm{g} / \mathrm{ml}$ mixture of penicillin and streptomycin (Lonza, Walkersville, MD, USA). Human SK5MEL cells were obtained from the American Type Culture Collection (ATCC; Manassas, VA, USA) and cultured in Eagle's minimum essential medium (ATCC) supplemented with $10 \%$ FBS and $100 \mu \mathrm{g} /$ $\mathrm{ml}$ mixture of penicillin and streptomycin.

Reverse transcription-quantitative PCR $(R T-q P C R)$. The mRNA expression of PAF-R was analyzed in the human SK5MEL and SK23MEL cells using RT-qPCR and the expression levels were normalized with GAPDH, as described previously $(8,13)$. The B16-PAF-R and B16-MSCV cells were used as positive and negative controls. Briefly, the cells were homogenized using an RLT buffer containing $\beta$-mercaptoethanol (Sigma-Aldrich), in a bullet blender (Next Advance, Inc., Averill Park, NY, USA) and carbide beads. The total RNA was extracted using an RNAeasy kit according to the manufacturer's instructions. The purified RNA was quantified using a Nano Drop 2000 (Thermo Fisher Scientific, Inc., Lafayette, CO, USA) and reverse transcribed with a Super Script cDNA synthesis kit containing random hexamers. The cDNA was analyzed for the PAF-R mRNA using a SYBR green-based, quantitative fluorescent PCR method $(6-8,13)$. The fluorescence was detected using a Step One Real-time PCR machine (Applied Biosystems, Foster City, CA, USA). The quantification of each PCR product was normalized to GAPDH using the $2^{-\Delta \Delta C t}$ method.

Cell proliferation. The B16-PAF-R and B16-MSCV cells were plated in 24-well plates (20,000 cells/well) and treated with 1 or $10 \mathrm{nM}$ 1-hexadecyl-2-N-methylcarbamoyl-3-glycerophosphocholine (CPAF) and incubated for 24, 48 or $72 \mathrm{~h}$. The control cells received $0.1 \%$ ethanol dissolved in phosphate-buffered saline (PBS;10 $\mu \mathrm{l}$ ) only. Following each time point, the cells were trypsinized, washed, resuspended in PBS and stained using $0.4 \%$ trypan blue according to the manufacturer's instructions (Invitrogen Life Technologies). The cell proliferation was assessed using a trypan blue exclusion method and a Countess automated cell counter (Invitrogen Life Technologies).

Cell survival. The murine or human melanoma cells were seeded into 96 -well plates $(5,000$ cells/well) and treated with various concentrations of BITC, as indicated in respective figures and figure legends. The control cells received $0.1 \%$ dimethylsulfoxide (DMSO) treatment. The cell survival rate was assessed $24 \mathrm{~h}$ after treatment using a sulforhodamine-B (SRB) assay, as described previously (19). The plates were read at 590nm using a Bio Kinetics plate reader (EL-800; BioTek Instruments, Inc., Winooski, VT, USA).

Reactive oxygen species (ROS) generation. The B16-MSCV and B16-PAF-R cells $\left(1 \times 10^{5}\right.$ cells/well) were seeded into 6 -well plates for attachment overnight. The cells were treated with H2DCFDA dye (DFC; $5 \mu \mathrm{M})$ for $30 \mathrm{~min}$ prior to treatment with $2 \mu \mathrm{M}$ BITC for $0,5,10 \mathrm{~min}$ or $1 \mathrm{~h}$. In a separate experiment, the cells were pre-treated with vitamin $\mathrm{C}(5 \mathrm{mM})$ for $1 \mathrm{~h}$ prior to treatment with $2 \mu \mathrm{M}$ BITC for $10 \mathrm{~min}$. The control cells received $0.1 \%$ DMSO treatment. The generation of ROS was analyzed by measuring the number of DCF-positive cells using a flow associated cell sorter and FlowJo software version 9.7.5 (Tree Star, Inc., Ashland, OR, USA).

Apoptosis. The B16-PAF-R and B16-MSCV cells (1x10 $0^{5}$ cells/well) were seeded into 6-well plates for attachment overnight. The cells were then either pretreated with $5 \mathrm{mM}$ vitamin $\mathrm{C}$ or $10 \mu \mathrm{M}$ WEB2086 PAF-R antagonist, for $1 \mathrm{~h}$, followed by treatment with $2 \mu \mathrm{M}$ BITC for $24 \mathrm{~h}$. The control cells received $0.1 \%$ DMSO treatment. Apoptosis was quantified using a luminescence caspase-3/7 glo-assay kit and normalized against the total protein, as described previously (28). The protein content of the samples were determined using a Bradford assay kit (Bio-Rad Laboratories, Inc., Hercules, CA, USA).

Statistical analysis. Each data set is representative of the combined results of at least three independent experiments with similar findings. Data were analyzed using GraphPad Prism software version 5 (GraphPad software, San Diego, CA, USA). Student's t-test and one-way analysis of variance with a Bonferroni Post-hoc test were used to compare two groups or more than two groups. $\mathrm{P}<0.05$ was considered to indicate a statistically significant difference.

\section{Results}

Evaluation of the expression of PAF-R and the effect of $C P A F$ the PAF-R agonist on melanoma cell growth. The majority of human melanoma cells express PAF-R, however, the B16F10 murine melanoma cell line, does not $(9,13)$. To determine 
A

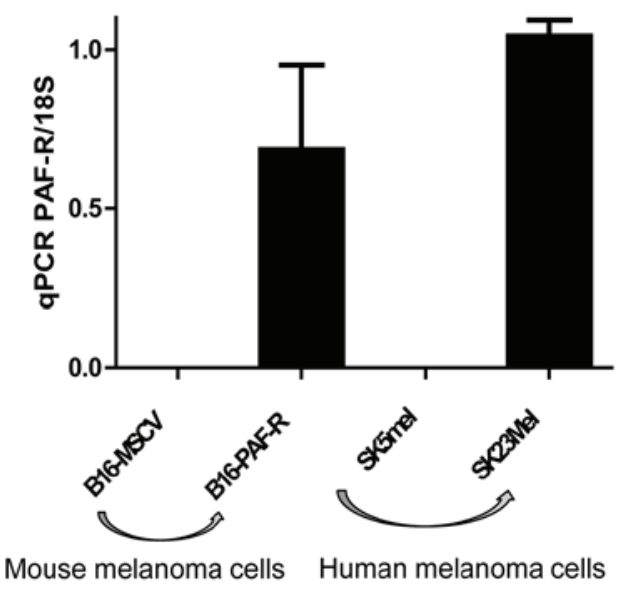

B

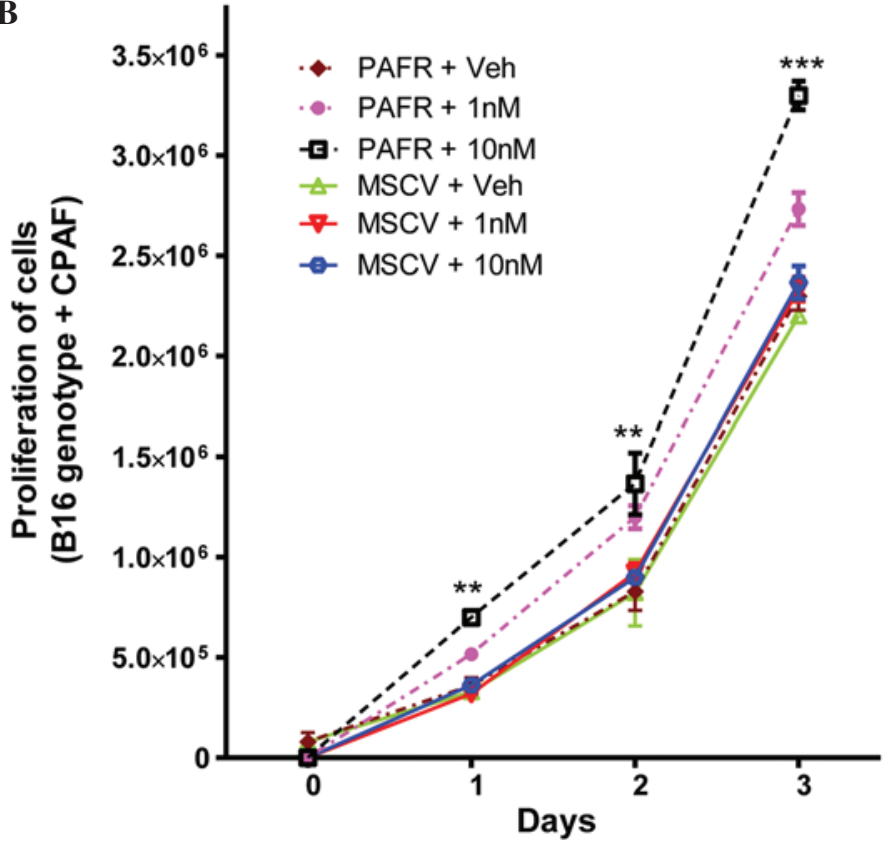

Figure 1. Effect of the PAF-R status on the proliferation of melanoma cells. (A) Evaluation of the presence of PAF-R status using reverse transcription-qPCR in murine and human melanoma cells. (B) B16-PAF-R and B16-MSCV cells were seeded into 24-well plates (20,000 cells/well) and allowed to attach overnight. The cells were treated with either vehicle or 1 or 10nM CPAF PAF-R agonist and cultured for 1, 2 or 3 days. At each time point, the cells were trypsinized and the number of cells were counted using a trypan blue exclusion method. Data are expressed as the mean \pm standard deviation of the cell proliferation by CPAF over the number of days. Statistically significant differences were observed between the B16-MSCV and B16-PAF-R cells at 10nM CPAF on days 1,2 and $3\left({ }^{* * *} \mathrm{P}<0.001\right.$ and ${ }^{* *} \mathrm{P}<0.01$ vs. MSCV $+10 \mathrm{nM}$ group). No significant differences were observed in the baseline growth rate between the B16-MSCV and B16-PAF-R cells. PAF-R, platelet-activating factor-receptor; MSCV, empty vector; qPCR, quantitative polymerase chain reaction; CPAF, 1-hexadecyl-2-N-methylcarbamoyl-3-glycerophosphocholine, VEH, vehicle.

the role of the PAF-R, a B16-PAF-R cell line was created by transduction of PAF-R-negative B16F10 cells with the MSCV2.1 retrovirus encoding the human leukocyte PAF-R, as described previously (13). B16-MSCV cells were used as a control. The human melanoma cells were then screened for the presence of functional PAF-R. RT-qPCR revealed the presence of functional PAF-R in the human SK23MEL cells (Fig. 1A). By contrast, human melanoma SK5MEL cells deficient in PAF-R were selected and used as a control (Fig. 1A). To evaluate the role of PAF-R in the progression of melanoma, the B16-PAF-R and B16-MSCV cells were treated with the non-metabolizable PAF-R agonist, CPAF, at different doses and time points prior to the assessment of cell proliferation. As shown in Fig. 1B, CPAF-treatment induced the proliferation of B16-PAF-R cells in a dose- and time-dependent manner, compared with the vehicle-treated B16-PAF-R cells. Notably, CPAF had no effect on the proliferation of the B16-MSCV cells at these doses, suggesting that PAF-R was involved in melanoma cell growth.

Effect of the BITC-mediated cytotoxicity of melanoma cells with regards to the PAF-R. Previous studies have demonstrated that ITC analogs, including BITC, exhibit decreased cytotoxicity towards melanoma cells in vitro (half maximal inhibitory concentration $10-20 \mu \mathrm{M}$ ), indicating that higher concentrations are required to achieve efficacy (29). The present study aimed to determine the effect of PAF-R on the BITC-mediated cytotoxicity of melanoma cells. The relative cytotoxicity of BITC in the melanoma cells was compared in the presence or absence of functional PAF-R using murine and human melanoma cells. PAF-R positive, B16-PAF-R and SK23MEL and PAF-R negative, B16-MSCV and SK5MEL cells were treated with different concentrations of BITC for $24 \mathrm{~h}$ and cell survival was measured. As shown in Fig. 2A and B, BITC dose-dependently reduced the survival of these cells. However, the expression of the PAF-R augmented the BITC-induced cytotoxicity in the B16-PAF-R ( $\left.\mathrm{IC}_{50} \sim 2 \mathrm{mM}\right)$ and SK23MEL $\left(\mathrm{IC}_{50} \sim 6 \mathrm{mM}\right)$ cells, compared with the PAF-R deficient cells. The $\mathrm{IC}_{50}$ of BITC in PAF-R deficient murine B16-MSCV cells was $\sim 11 \mathrm{mM}$ and in the human SK5MEL cells was $>50 \mu \mathrm{M}$. These findings indicated that PAF-R signaling augments the decreased survival of the melanoma cells, which is elicited by BITC, at a lower concentration than is required for the PAF-R-deficient cells.

BITC treatment enhances the generation of ROS in PAF-R-expressing melanoma cells. BITC acts as a pro-oxidative stressor, inducing the generation of ROS as a potent mechanism of tumor cell death $(21,22,24,30-32)$. By contrast, other studies have demonstrated that BITC can also mediate potent antioxidant effects against oxidized low density lipoprotein-induced endothelial dysfunction (33) and inflammation-mediated carcinogenesis $(34,35)$. To determine the mechanism underlying the BITC-induced decreased survival rate of the PAF-R expressing melanoma cells, the effect of BITC on ROS generation was measured. For mechanistic studies, B16-PAF-R and B16-MSCV cells were used as these lines were generated from the same parent $(\mathrm{B} 16 \mathrm{~F} 10)$ cells. As the $\mathrm{IC}_{50}$ of BITC in the B16-PAF-R cells was $\sim 2 \mu \mathrm{M}$, 
A

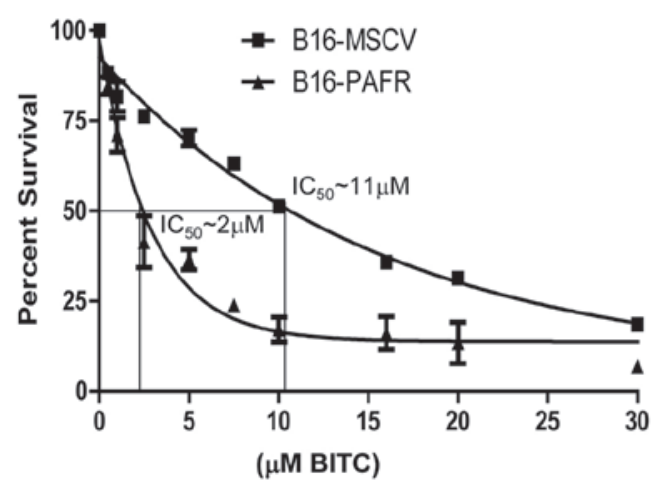

B

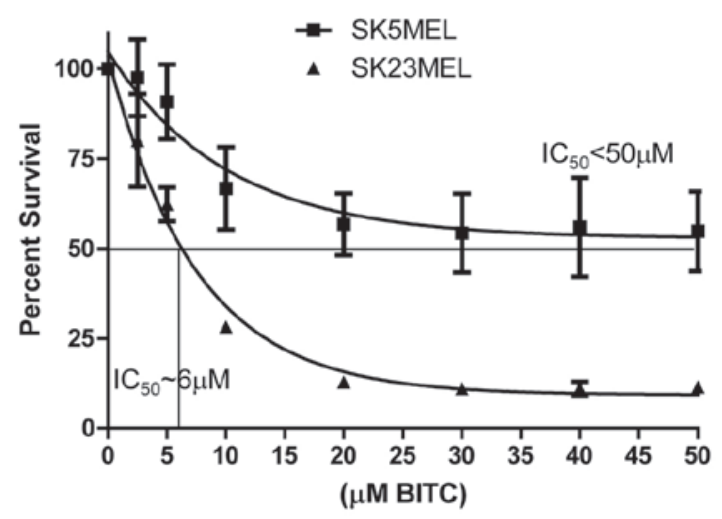

Figure 2. Effect of the expression of PAF-R on the BITC-mediated suppression of murine and human melanoma cell survival. (A and B) B16-PAF-R, B16-MSCV, SK23MEL and SK5MEL cells were treated with either vehicle (0.1\% DMSO) or different concentrations of BITC in vitro and incubated for $24 \mathrm{~h}$. The cell survival was measured following incubation using an sulforhodamine-B assay. Data are expressed as the mean \pm standard deviation and are presented as the percent survival against the BITC treatments. MSCV, empty vector; PAF-R, platelet-activating factor-receptor; BITC, benzyl isothiocyanate; DMSO, dimethylsulfoxide; $\mathrm{IC}_{50}$, half maximal inhibitory concentration.

A

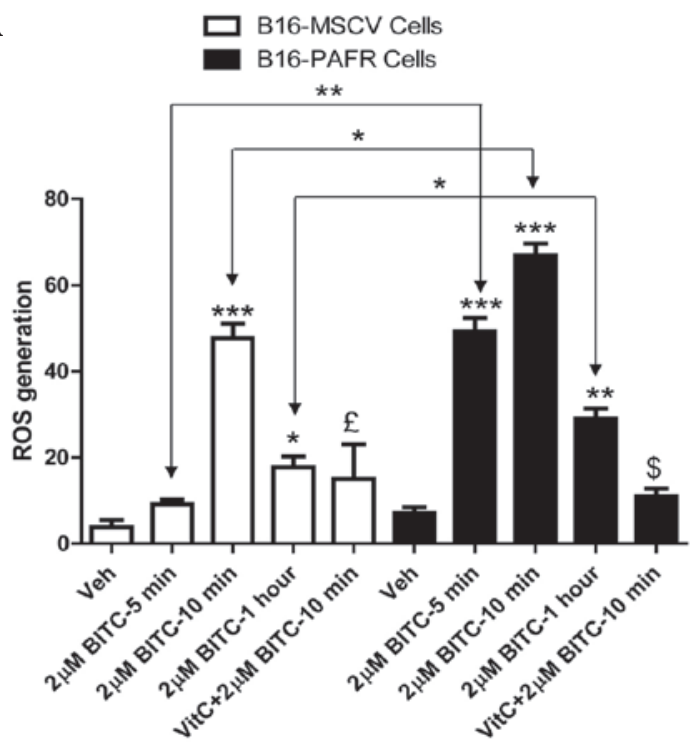

B

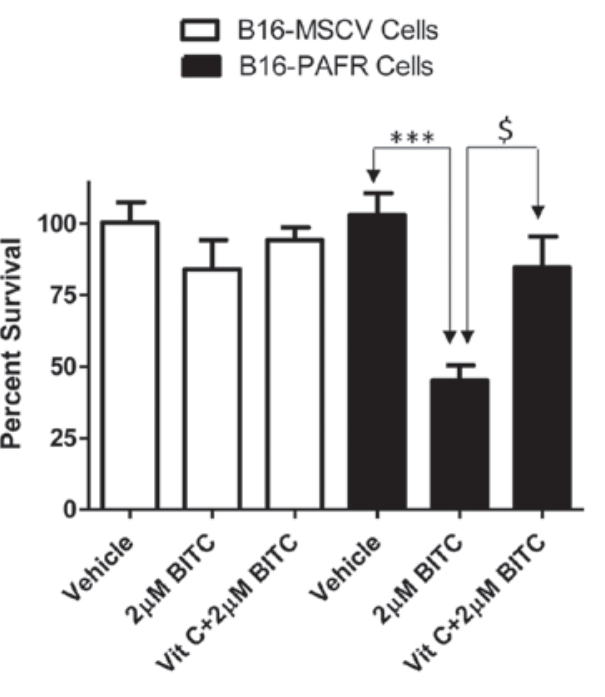

Figure 3. Effect of BITC on the generation of ROS in melanoma cells (A) Effect of BITC treatment $(2 \mu \mathrm{M})$ on the generation of ROS in the presence and absence of Vit C $(5 \mathrm{mM})$ was analyzed by measuring DCF fluorescence by flow cytometry. Data are represented as the mean \pm standard deviation and are presented as the generation of ROS against the different treatment groups. Statistically significant differences were observed between the vehicle and BITC treatment groups and the BITC and vit $\mathrm{C}+\mathrm{BITC}$ groups $\left({ }^{*} \mathrm{P}<0.05 ;{ }^{\circledR} \mathrm{P}<0.01,{ }^{\$} \mathrm{P}<0.001,{ }^{* *} \mathrm{P}<0.01\right.$ and $\left.{ }^{* * *} \mathrm{P}<0.001\right)$. (B) The effect of $\mathrm{BITC}$ on cell survival rates in the presence and absence of vit $\mathrm{C}(5 \mathrm{mM})$ in the B16-PAF-R and B16-MSCV cells was determined using an SRB assay. Data are expressed as the mean \pm standard deviation and presented as the percent survival against the different treatment groups. Statistically significant differences were observed between the vehicle and the BITC treated groups and the BITC and vitamin $\mathrm{C}+\mathrm{BITC}$ treated group $\left({ }^{* * * *} \mathrm{P}<0.001 ;{ }^{\$} \mathrm{P}<0.001\right)$. The control cells received vehicle (DMSO) treatment only. Veh, vehicle; BITC, benzyl isothiocyanate; MSCV, empty vector; PAF-R, platelet-activating factor-receptor; ROS, reactive oxygen species; Vit C, vitamin C.

this concentration of BITC was used to treat the B16-PAF-R and B16-MSCV cells at different time points. The cells were pretreated with the antioxidant, vitamin $\mathrm{C}(5 \mathrm{mM})$ for $1 \mathrm{~h}$ and subsequently with BITC. As shown in Fig. 3A, BITC treatment induced a significant increase in ROS generation in each of the cell lines. However, in the B16-PAF-R cells, ROS generation occurred as early as 5 min after treatment and was significantly increased compared with the B16-MSCV cells at all time points (Fig. 3A). Treatment with vitamin $\mathrm{C}$ inhibited the BITC-induced ROS generation (Fig. 3A) and rescued B16-PAF-R cells (Fig. 3B), indicating a role for ROS in the BITC-induced suppression of the B16-PAF-R cells.
PAF-R augments BITC-induced apoptosis in the B16-PAF-R cells. The induction of apoptosis in malignant cells has been revealed as a major mechanism of chemopreventive/therapeutic drug-mediated cell death $(12,15,19,30-33)$. To determine whether the expression of PAF-R augments the BITC-mediated decrease in B16-PAF-R cell survival via ROS generation, apoptosis induction was measured. The B16-PAF-R and B16-MSCV cells were treated with $2 \mu \mathrm{M}$ BITC for $24 \mathrm{~h}$ in the presence or absence of either vitamin C or the WEB2086 PAF-R antagonist. As shown in Fig. 4, BITC-treatment resulted in a significant apoptotic response in the B16-PAF-R cells compared with the B16-MSCV cells. The induction of apoptosis in the B16-PAF-R 


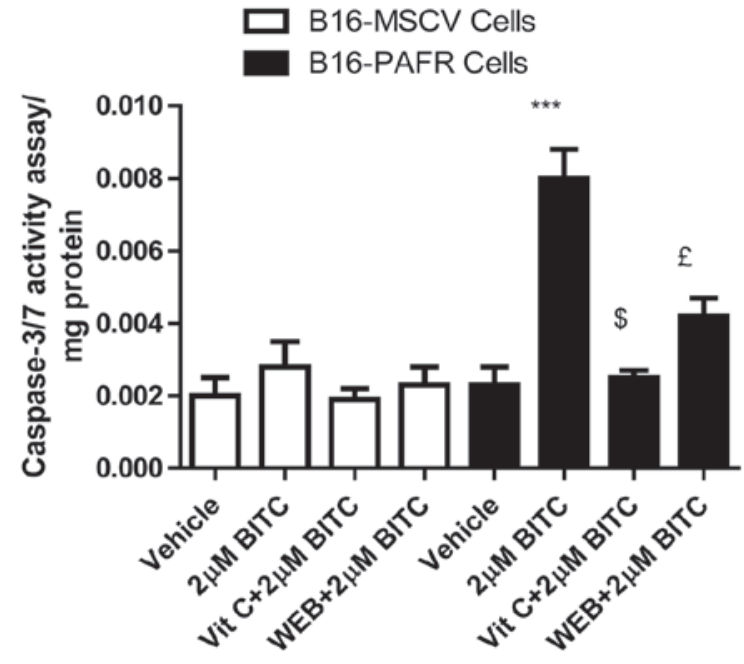

Figure 4. Effect of PAF-R on BITC-mediated apoptosis in the melanoma cells. The B16-MSCV and B16-PAF-R cells were pre-treated either with Vit $\mathrm{C}(5 \mathrm{mM})$ or the WEB2086 PAF-R antagonist $(10 \mu \mathrm{M})$, for $1 \mathrm{~h}$ followed by treatment with $2 \mu \mathrm{M}$ BITC for $24 \mathrm{~h}$. Apoptosis was assessed using a fluorogenic caspase-3/7 activity assay kit. Data are expressed as the mean \pm standard deviation and are presented as caspase- $3 / 7$ activity $/ \mathrm{mg}$ protein against the different treatment groups. Statistically significant differences were observed between the vehicle and BITC, BITC and Vitamin C + BITC and BITC and WEB + BITC $\left({ }^{* * *} \mathrm{P}<0.001\right.$ vs. vehicle group; ${ }^{\mathrm{S}} \mathrm{P}<0.001$ vs. $2 \mu \mathrm{M}$ BITC group and ${ }^{\mathfrak{f}} \mathrm{P}<0.01$ vs. $2 \mu \mathrm{M}$ BITC group). PAF-R, platelet-activating factor-receptor; BITC, benzyl isothiocyanate; MSCV, empty-vector; Vit C, vitamin C; WEB, WEB2086.

cells was significantly attenuated following treatment with vitamin C and WEB2086. These results indicated the involvement of PAF-R signaling in the BITC-induced decrease in growth of the B16-PAF-R cells mediated via increased ROS generation and induction of apoptosis.

\section{Discussion}

The activation of PAF-R is important in diverse biological processes, including regulating the growth of melanoma (3-13), and the majority of types of melanoma express PAF-R (9) and are resistant to the currently used chemotherapeutic agents (16). Therefore, the present study investigated the efficacy of BITC against melanoma cells, in those either expressing PAF-R or not. Human melanoma cells, either deficient in or expressing PAF-R, and murine melanoma B16F10 cells, which have been extensively used in chemotherapy studies and lack functional PAF-R expression, were genetically modified to produce cells stably expressing PAF-R or vector-controls as suitable model systems.

Treatment with CPAF increased the proliferation of the PAF-R-expressing B16-PAF-R cells in a dose- and time-dependent manner compared with the B16-MSCV cells, confirming that PAF-R activation enhances the growth of melanoma cells. By contrast, BITC-treatment reduced the survival rate of the murine and human melanoma cells in a dose-dependent manner. However, the presence of functional PAF-R potentiated the cytotoxicity of BITC in the B16-PAF-R murine and human SK23MEL melanoma cells, requiring relatively lower doses than were required for the PAF-R deficient cells. This indicated that the expression of PAF-R results in enhanced cytotoxicity of the melanoma cells by BITC.
Generation of ROS is an early event following chemotherapy, that is important in inducing apoptosis in malignant cells (15,30-32). In this context, treatment with BITC at doses that substantially reduced the survival of the B16-PAF-R cells, increased the generation of ROS and the levels of apoptosis compared with the B16-MSCV cells. These effects were attenuated by vitamin $\mathrm{C}$, suggesting the involvement of ROS in the BITC-induced decreased survival of B16-PAF-R cells. These data are consistent with previous studies, which demonstrated that the BITC-induced suppression of malignant cells was mediated via ROS generation and inhibited by free radical quenchers (30). The present study demonstrated that BITC-treatment resulted in an increased level of apoptosis of the B16-PAF-R cells via ROS and confirmed the role of the PAF-R activation. The BITC-induced increase in apoptosis in the B16-PAF-R cells was inhibited by the WEB2086 PAF-R antagonist, confirming the involvement of PAF-R signaling in this process. The ability of PAF-R signaling to promote the proliferation of the B16-PAF-R cells and to augment BITC-mediated apoptosis was in agreement with previous studies, which observed that CPAF-treatment induced increased proliferation in a PAF-R-negative KB epithelial cell line, which was genetically modified to stably express the functional PAF-R (KBP), however this did not occur in retroviral vector-transduced control KBM cells (36). Similarly, the expression of PAF-R augmented ultraviolet B (UVB)-mediated apoptosis of PAF-R-positive KBP but not PAF-R-negative KBM cells (14). This increased susceptibility of the KBP cells towards UVB-mediated enhanced apoptosis was inhibited by antioxidants and PAF-R antagonists. Additionally, PAF-R activation augmented chemotherapy-induced cytotoxicity in human carcinoma cell lines (15). These previous studies confirm the involvement of the PAF-R signaling in mediating pro-oxidative stressors including BITC-mediated increase in melanoma cell apoptosis.

In conclusion, naturally occurring ITCs have been demonstrated to possess anticarcinogenic properties, however, the lack of specific oncogenic targets and the use of higher concentrations to achieve optimum therapeutic efficacy have made them unsuitable. Therefore, the selection of potent analogs, which can target specific signaling pathways may offer more effective agents against malignant cells. The present study demonstrated that BITC suppressed the growth of melanoma cells and that this was augmented by the activation of PAF-R through the ROS-mediated pathway. Collectively, these data suggest that BITC may be used as a novel chemotherapeutic agent against PAF-R-expressing melanoma cells.

\section{Acknowledgements}

The present study was supported by a grant from the American Institute for Cancer Research (no. 09A062), the American Cancer Society-Institutional Research Grant (no. 4185607), the Showalter Research Trust Award (no. 4485602) and the National Institute of Health K22 (no. ES0238850). The authors would like to thank Dr Christopher Touloukian from the Department of Surgery, Indiana University School of Medicine (Indianapolis, USA) for the human melanoma SK23MEL cells (37), Dr Jeffrey Travers (Department of Dermatology, Indiana University School of Medicine, 
Indianapolis, IN 46202, USA) and Dr Raymond Konger (Laboratory Medicine and Dermatology, Indiana University School of Medicine, Indianapolis, IN 46202, USA) for their support and critically evaluating this manuscript. The abstract was published as abstract no. LB332 in FASEBJ 28 (Suppl 1), 2014.

\section{References}

1. Miller AJ and Mihm MC Jr: Melanoma. N Engl J Med 355: 51-65, 2006

2. National Cancer Institute: SEER Stat Fact Sheets: Melanoma of the Skin. http://seer.cancer.gov/statfacts/html/melan.html. Accessed January 21, 2015.

3. Ishii S, Nagase T and Shimizu T: Platelet-activating factor receptor. Prostaglandins Other Lipid Mediat 68: 599-609, 2002.

4. Zhuang Q, Bastien Y and Mazer BD: Activation via multiple signaling pathways induces down-regulation of platelet-activating factor receptors on human B lymphocytes. J Immunol 165 2423-2431, 2000.

5. Travers JB,HuffJC,Rola-Pleszczynski M,GelfandEW,MorelliJG and Murphy RC: Identification of functional platelet-activating factor receptors on human keratinocytes. J Invest Dermatol 105: 816-823, 1995.

6. Sahu RP, Kozman AA, Yao Y, DaSilva SC, Rezania S, Martel KC, Warren SJ, Travers JB and Konger RL: Loss of the platelet activating factor receptor in mice augments PMA-induced inflammation and cutaneous chemical carcinogenesis. Carcinogenesis 33: 694-701, 2012.

7. Sahu RP, Petrache I, Van Demark MJ, Rashid BM, Ocana JA, Tang Y, Yi Q, Turner MJ, Konger RL and Travers JB: Cigarette smoke exposure inhibits contact hypersensitivity via the generation of platelet-activating factor agonists. J Immunol 190: 2447-2454, 2013.

8. Hackler PC, Reuss S, Konger RL, Travers JB and Sahu RP: Systemic platelet-activating factor receptor activation augments experimental lung tumor growth and metastasis. Cancer Growth Metastasis 19: 27-32, 2014.

9. Melnikova VO, Mourad-Zeidan AA, Lev DC and Bar-Eli M: Platelet-activating factor mediates MMP-2 expression and activation via phosphorylation of cAMP-response element-binding protein and contributes to melanoma metastasis. J Biol Chem 281 2911-2922, 2006.

10. Heon Seo K, Ko HM, Kim HA, Choi JH, Jun Park S, Kim KJ, Lee HK and Im SY: Platelet-activating factor induces up-regulation of antiapoptotic factors in a melanoma cell line through nuclear factor-kappaB activation. Cancer Res 66: 4681-4686, 2006.

11. Biancone L, Cantaluppi V, Del Sorbo L, Russo S, Tjoelker LW, and Camussi G: Platelet-activating factor inactivation by local expression of platelet-activating factor acetyl-hydrolase modifies tumor vascularization and growth. Clin Cancer Res 9: 4214-4220, 2003.

12. de Oliveira SI, Andrade LN, Onuchic AC, Nonogaki S, Fernandes PD, Pinheiro MC, Rohde CB, Chammas R and Jancar S: Platelet-activating factor receptor (PAF-R)-dependent pathways control tumour growth and tumour response to chemotherapy. BMC Cancer 10: 200, 2010.

13. Sahu RP, Turner MJ, DaSilva SC, Rashid BM, Ocana JA, Perkins SM, Konger RL, Touloukian CE, Kaplan MH and Travers JB: The environmental stressor ultraviolet B radiation inhibits murine antitumor immunity through its ability to generate platelet-activating factor agonists. Carcinogenesis 33: 1360-1367, 2012

14. Barber LA, Spandau DF, Rathman SC, Murphy RC, Johnson CA, Kelley SW, Hurwitz SA, Travers JB: Expression of the platelet-activating factor receptor results in enhanced ultraviolet $\mathrm{B}$ radiation-induced apoptosis in a human epidermal cell line. J Biol Chem 273: 18891-18897, 1998.

15. Li T, Southall MD, Yi Q, Pei Y, Lewis D, Al-Hassani M, Spandau D and Travers JB: The epidermal platelet-activating factor receptor augments chemotherapy-induced apoptosis in human carcinoma cell lines. J Biol Chem 278: 16614-16621, 2003.

16. Jilaveanu LB, Aziz SA and Kluger HM: Chemotherapy and biologic therapies for melanoma: Do they work? Clin Dermatol 27: 614-625, 2009.
17. Block G, Patterson B and Subar A: Fruit, vegetables and cancer prevention: A review of the epidemiological evidence. Nutr Cancer 18: 1-29, 1992.

18. Stoner GD and Morse MA: Isothiocyanates and plant polyphenols as inhibitors of lung and esophageal cancer. Cancer Lett 114: 113-119, 1997.

19. Sahu RP, and Srivastava SK: The role of STAT-3 in the induction of apoptosis in pancreatic cancer cells by benzyl isothiocyanate. J Natl Cancer Inst 101: 176-193, 2009.

20. Warin R, Xiao D, Arlotti JA, Bommareddy A and Singh SV: Inhibition of human breast cancer xenograft growth by cruciferous vegetable constituent benzyl isothiocyanate. Mol Carcinog 49: 500-507, 2010.

21. Wu CL, Huang AC, Yang JS, Liao CL, Lu HF, Chou ST, Ma CY, Hsia TC, Ko YC and Chung JG: Benzyl isothiocyanate (BITC) and phenethyl isothiocyanate (PEITC)-mediated generation of reactive oxygen species causes cell cycle arrest and induces apoptosis via activation of caspase-3, mitochondria dysfunction and nitric oxide (NO) in human osteogenic sarcoma U-2 OS cells. J Orthop Res 29: 1199-1209, 2011.

22. Wu X, Zhu Y, Yan H, Liu B, Li Y, Zhou Q and Xu K: Isothiocyanates induce oxidative stress and suppress the metastasis potential of human non-small cell lung cancer cells. BMC Cancer 10: 269,2010.

23. Ni WY, Hsiao YP, Hsu SC, Hsueh SC, Chang CH, Ji BC, Yang JS, Lu HF and Chung JG. Oral administration of benzyl-isothiocyanate inhibits in vivo growth of subcutaneous xenograft tumors of human malignant melanoma A375.S2 cells. In Vivo 27, 623-626, 2013.

24. Huang SH, Wu LW, Huang AC, Yu CC, Lien JC, Huang YP, Yang JS, Yang JH, Hsiao YP, Wood WG, Yu CS and Chung JG: Benzyl isothiocyanate (BITC) induces $\mathrm{G} 2 / \mathrm{M}$ phase arrest and apoptosis in human melanoma A375.S2 cells through reactive oxygen species (ROS) and both mitochondria-dependent and death receptor-mediated multiple signaling pathways. J Agric Food Chem 60: 665-675, 2012.

25. Thejass P and Kuttan G: Allyl isothiocyanate (AITC) and phenyl isothiocyanate (PITC) inhibit tumour-specific angiogenesis by downregulating nitric oxide (NO) and tumour necrosis factor-alpha (TNF-alpha) production. Nitric Oxide 16: 247-257, 2007.

26. Manesh C and Kuttan G: Effect of naturally occurring allyl and phenyl isothiocyanates in the inhibition of experimental pulmonary metastasis induced by B16F-10 melanoma cells. Fitoterapia 74: 355-363, 2003

27. Hamsa TP, Thejass P and Kuttan G: Induction of apoptosis by sulforaphane in highly metastatic B16F-10 melanoma cells. Drug Chem Toxicol 34: 332-340, 2011.

28. Sahu RP, DaSilva SC, Rashid B, Martel KC, Jernigan D, Mehta SR, Mohamed DR, Rezania S, Bradish JR, Armstrong $\mathrm{AB}$, Warren $\mathrm{S}$ and Konger RL: Mice lacking epidermal PPAR $\gamma$ exhibit a marked augmentation in photocarcinogenesis associated with increased UVB-induced apoptosis, inflammation and barrier dysfunction. Int J Cancer 131: E1055-E1066, 2012.

29. Sharma A, Sharma AK, Madhunapantula SV, Desai D, Huh SJ, Mosca P, Amin S and Robertson GP: Targeting Akt3 signaling in malignant melanoma using isoselenocyanates. Clin Cancer Res 15: 1674-1685, 2009.

30. Sahu RP, Zhang R, Batra S, Shi Y and Srivastava SK. Benzyl isothiocyanate-mediated generation of reactive oxygen species causes cell cycle arrest and induces apoptosis via activation of MAPK in human pancreatic cancer cells. Carcinogenesis 30: 1744-1753, 2009

31. Xiao D, Powolny AA and Singh SV: Benzyl isothiocyanate targets mitochondrial respiratory chain to trigger reactive oxygen species-dependent apoptosis in human breast cancer cells. J Biol Chem 283: 30151-30163, 2008.

32. Miyoshi N, Watanabe E, Osawa T, Okuhira M, Murata Y, Ohshima $\mathrm{H}$ and Nakamura Y: ATP depletion alters the mode of cell death induced by benzyl isothiocyanate. Biochim Biophys Acta 1782: 566-573, 2008.

33. Doroshow JH: Redox modulation of chemotherapy-induced tumor cell killing and normal tissue toxicity. J Natl Cancer Inst 98: 223-235, 2006.

34. Huang CS, Lin AH, Liu CT, Tsai CW, Chang IS, Chen HW and Lii CK: Isothiocyanates protect against oxidized LDL-induced endothelial dysfunction by upregulating Nrf2-dependent antioxidation and suppressing NFKB activation. Mol Nutr Food Res 57: 1918-1930, 2013 
35. Miyoshi N, Takabayashi S, Osawa T and Nakamura Y: Benzyl isothiocyanate inhibits excessive superoxide generation in inflammatory leukocytes: implication for prevention against inflammation-related carcinogenesis. Carcinogenesis 25: 567-575 2004.

36. Marques SA, Dy LC, Southall MD, Yi Q, Smietana E, Kapur R, Marques M, Travers JB and Spandau DF: The platelet-activating factor receptor activates the extracellular signal-regulated kinase mitogen-activated protein kinase and induces proliferation of epidermal cells through an epidermal growth factor-receptor-dependent pathway. J Pharmacol Exp Ther 300: 1026-1035, 2002.
37. Brandmaier AG, Leitner WW, Ha SP, Sidney J, Restifo NP and Touloukian CE: High-avidity autoreactive CD4+ T cells induce host CTL, overcome T(regs) and mediate tumor destruction. J Immunother 32: 677-688, 2009. 